\title{
Treating Persistent Ectopic Pregnancy by Methotrexate Using a Sliding Scale: Preliminary Experience
}

\author{
MARK V. SAUER, M.D., ${ }^{1}$ ANDREA VIDALI, M.D., ${ }^{1}$ and WALTER JAMES, M.D. ${ }^{2}$
}

\begin{abstract}
To assess the clinical utility of prescribing a sliding scale regimen for methotrexate administered in cases of persistent ectopic pregnancy, a prospectively designed clinical trial was performed. Patients $(n=37)$ previously operated on for ectopic pregnancy by laparoscopic salpingostomy and found to have persistent disease were enrolled into the study. Methotrexate was given intramuscularly at a dose of $1 \mathrm{mg} / \mathrm{kg}$ body weight, with the number of doses administered depending on the presenting level of serum beta-human chorionic gonadotropin $(\beta$-hCG). Group $1(n=27)$, levels of $10-1500 \mathrm{mIU} / \mathrm{ml}$, received a single dose; group $2(n=$ 5), levels of $1500-2500 \mathrm{mIU} / \mathrm{ml}$, received two doses 3 days apart; group $3(n=5)$, levels $>2500 \mathrm{mIU} / \mathrm{ml}$, received three doses 3 days apart. Patients were followed with weekly transvaginal ultrasound examinations, and serum $\beta$-hCG and progesterone measurements. Results noted resolution in all three treatment groups without the requirement of surgery or further treatment: group 1, $19.9 \pm 1.7$ days; group $2,35.2 \pm 4.5$ days; group 3, $49.0 \pm 1.0$ days. All patients remained asymptomatic, and no adverse side effects from the drug were experienced. We conclude, although preliminary, that a sliding scale approach to prescribing methotrexate based on the initial serum titer of $\beta$-hCG to treat persistent ectopic pregnancy is easy to use, efficacious, and likely results in complete resolution of the condition. In most cases, even with presenting levels of $\beta$-hCG $>2500 \mathrm{mIU} / \mathrm{ml}$, persistent disease will resolve within 50 days of initiating care. (J GYNECOL SURG 13:13, 1997)
\end{abstract}

\section{INTRODUCTION}

$\mathbf{T}$ HE DIAGNOSIS AND SURGICAL TREATMENT of ectopic pregnancy have been accomplished increasingly through the laparoscope. Most tubal gestations, whether ruptured or unruptured, may be successfully and quickly evacuated through a salpingostomy and removed through a second site port placed in the lower abdomen. ${ }^{1}$ This approach obviates the need for performing a laparotomy and reduces surgical morbidity and patient recovery time. ${ }^{2}$ Therefore, when it can be safely attempted, the laparoscopic approach optimizes patient care. ${ }^{3}$

Despite efforts at completely eradicating the ectopic pregnancy during surgical extirpation, persistent trophoblast proliferation occurs in 5\%-10\% of cases when a salpingostomy is performed. ${ }^{4,5}$ Unfortunately, if left untreated, occasional patients may later rupture their fallopian tube and suffer catastrophic hemorrhage. ${ }^{6}$ Therefore, once persistent cases are diagnosed, it is best to either reoperate and remove the affected site or administer methotrexate to destroy the persistent gestation. ${ }^{7}$

${ }^{1}$ Columbia University College of Physicians and Surgeons, New York, New York.

${ }^{2}$ Department of Obstetrics and Gynecology, University of Southern California School of Medicine, Los Angeles, California. 
There is no consensus as to whether surgery or medicine is the better approach for treating persistent disease. Furthermore, no standard protocol exists for delivering methotrexate to patients with persistent ectopic pregnancy following evacuative surgery. Often, women are prescribed drugs in much the same manner as women receiving methotrexate as primary treatment for unruptured ectopic pregnancies. ${ }^{8}$ Thus, physicians are often confused as to which regimen to follow and how best to modify published protocols to suit the needs of the patient.

We devised a protocol for diagnosing and treating persistent cases of ectopic pregnancy based on patterns and titers of serum progesterone and beta-human chorionic gonadotropin $(\beta=\mathrm{hCG}) .{ }^{9}$ This simplified approach uses an easy to remember sliding scale regimen designed to minimize expensive and time-consuming follow-up visits while providing reasonable guidelines for continuing treatment. We report our preliminary experience.

\section{MATERIALS AND METHODS}

The use of methotrexate for the treatment of persistent ectopic pregnancy in women previously operated on was reviewed and approved by the Institutional Review Board of the University of Southern California, Los Angeles. During the course of the 24-month clinical trial, approximately 700 women were treated at the Los Angeles County-USC Medical Center for ectopic pregnancy. Patients who had recently undergone laparoscopic salpingostomy for ectopic pregnancy were followed postoperatively using serum measurements for $\beta$-hCG and progesterone until resolution $(\beta$-hCG $<5.0 \mathrm{mIU} / \mathrm{ml}$; progesterone $<0.5 \mathrm{ng} / \mathrm{ml})$. Patients in whom persistent disease was documented, as evidenced by elevations in both serum progesterone $(>1.5$ $\mathrm{ng} / \mathrm{ml})$ and $\beta$-hCG $(>0.1 \times$ level obtained preoperatively), by the tenth postoperative day were considered for treatment. ${ }^{9}$

Subjects $(n=37)$ were offered medical therapy using methotrexate $(1 \mathrm{mg} / \mathrm{kg}$ body weight $)$ instead of reoperation if they met the following entry criteria: asymptomatic, normal liver, renal, and hematologic studies, agreed to be compliant with follow-up visits, and were desirous of avoiding a second-look procedure. Transvaginal ultrasound examination of the pelvis was performed before initiating therapy to exclude adnexal pathology and guard against unknowingly treating a heterotopic intrauterine gestation. At the time of these scans, no patient demonstrated ultrasonographically visible signs of the ectopic sites.

Following informed consent, the women were given methotrexate as a single intramuscular injection. The dosage and injection schedule was based on the level of $\beta$-hCG exhibited at the time of study entry and were accordingly grouped. Group 1 subjects had levels of $10-1500 \mathrm{mIU} / \mathrm{ml}$ (International Reference Preparation) and were given a single dose. The range of values for these patients $(n=27)$ was $55-1450 \mathrm{mIU} / \mathrm{ml}$. Group $2(n=5)$ levels were $1500-2500 \mathrm{mIU} / \mathrm{ml}$ (range $1600-2450 \mathrm{mIU} / \mathrm{ml})$, and patients received a second dose intramuscularly 3 days later. Group 3 subjects $(n=5)$ had values $>2500 \mathrm{mIU} / \mathrm{ml}$ (range 2500-5500 $\mathrm{mIU} / \mathrm{ml}$ ) and received a third dose 3 days after the second dose, for a total of three doses. Liver function tests and complete blood counts with platelets were repeated before administering each dose. Weekly visits were scheduled to obtain levels of $\beta$-hCG and progesterone, as well as repeat transvaginal ultrasound examinations, in order to monitor the progress of subjects following treatment.

\section{RESULTS}

The women enrolled for study $(n=37)$ had all undergone linear salpingostomy performed laparoscopically by residents in obstetrics and gynecology within 3 weeks of coming for treatment. At diagnosis, all women were noted to have elevated serum progesterone (mean \pm standard error; $3.2 \pm 1.3 \mathrm{ng} / \mathrm{ml}$ ) and $\beta$ hCG levels. Table 1 lists the patients according to treatment group and shows the clinical outcomes.

All women were asymptomatic at the time of persistent proliferation and remained asymptomatic throughout the treatment and surveillance period. No patient required additional medications beyond that prescribed by protocol. For each group and in all cases, serum progesterone declined to $<0.5 \mathrm{ng} / \mathrm{ml}$ within 1 week of the final dose of methotrexate. In 9 of 10 women receiving more than a single dose of methotrexate, progesterone remained elevated $>1.5 \mathrm{ng} / \mathrm{ml}$ before their final injection, implying the need for the additional drug. Patient compliance was excellent, and all subjects returned for follow-up visits and blood tests. There 
Table 1. Clinical Characteristics of Patients with Persistent Ectopic Gestations Receiving Varying Doses of Methotrexate Administered by Sliding Scale

\begin{tabular}{lccr}
\hline & $\begin{array}{c}\beta-h C G \text { at diagnosis } \\
\text { Group }\end{array}$ & \multicolumn{2}{c}{ Days to resolution $(\beta-h C G<5 \mathrm{mIU} / \mathrm{ml})$} \\
\cline { 3 - 4 } $1(n=27)$ & $10-1500$ & Mean $\pm S E$ & Range \\
$2(n=5)$ & $1500-2500$ & $19.9 \pm 1.7$ & $5-42$ \\
$3(n=5)$ & $>2500$ & $35.2 \pm 4.5$ & $28-39$ \\
\hline
\end{tabular}

were no changes noted in hepatic, renal, or hematologic parameters, and no complications or side effects occurred during therapy.

\section{DISCUSSION}

The treatment of ectopic pregnancy has continued to evolve as diagnostic imaging and serum monitoring improve. The ability to discover tubal gestations earlier allows broadening of operative choices and lessens the need for performing laparotomy. Most cases can be handled laparoscopically, with salpingostomy usually chosen to preserve the tube and maximize fertility potential. This approach is relatively easy to teach, and residents typically learn basic pelviscopic surgery on patients with ectopic pregnancy. However, incomplete removal of the trophoblast occurs in 5\%-10\% of cases and necessitates a second procedure or an attempt at medical management. This figure may be even higher in centers with training programs. Thus, the need for aggressive postoperative surveillance is imperative to ensure good outcomes.

Treatment of persistent cases of ectopic pregnancy include salpingectomy, repeat salpingostomy, expectant management, and methotrexate therapy. ${ }^{10}$ In symptomatic patients or in instances in which a large pelvic mass is sonographically visualized, surgical intervention is indicated. However, this occurs in a minority of cases. Most patients will remain asymptomatic, and no visible proliferation of trophoblast is evident on bimanual or ultrasound examination. These women make excellent candidates for nonsurgical intervention, which avoids the expense and morbidity of a second operation.

The dose of methotrexate used in this study and its scheduled delivery were arbitrarily chosen. However, this regimen closely parallels the amount of drug needed to treat small unruptured ectopic pregnancies in our original clinical trial, when the medication was used as primary treatment. ${ }^{11}$ The mass of viable trophoblast producing $\beta$-hCG is proportional to its serum level, and often more than a single injection of methotrexate will be necessary. In cases of primary medical management, the more advanced gestations required the longest duration of treatment and the highest cumulative dose of drug.

The toxicity of methotrexate is primarily related to its duration of use. ${ }^{12}$ The doses administered in this trial are consistent with regimens prescribed to women with gestational trophoblastic disease and to protocols used in primary medical management of unruptured ectopic pregnancy. ${ }^{11}$ Complications are rare, most often being transient rises in liver enzyme values, and occur in under $10 \%$ of patients. Even these untoward events will be lessened by the proposed dosing schedule of every 3 days rather than consecutive-day delivery as was given in the past.

The use of a sliding scale should simplify implementing methotrexate and promote initiation of medical management of persistent disease. By design, this protocol addresses the need to treat the larger more viable trophoblastic masses aggressively yet delivers drug accordance to a schedule that minimizes toxicity. Prescribing methotrexate within a few weeks of surgery should increase patient compliance, as they are still in the recovery phase of treatment. The few follow-up visits required of medically managed patients greatly reduce the overall cost of care compared with reoperated patients whether or not outpatient surgery is used.

We conclude that the use of a standardized protocol for methotrexate prescribed according to a sliding scale scheduled format will adequately eradicate most, if not all, cases of persistent trophoblastic disease. A requisite for success is early diagnosis and immediate administration of the drug. 


\section{REFERENCES}

1. Silva PD. A laparoscopic approach can be applied to most cases of ectopic pregnancy. Obstet Gynecol 1988;72:944.

2. Vermesh M, Silva P, Rosen G, Stein A, Fossum G, Sauer M. Management of unruptured ectopic gestation by linear salpingostomy: A prospective, randomized clinical trial of laparoscopy versus laparotomy. Obstet Gynecol 1989;73:400.

3. Sauer MV, Rand T, Buster JE. Ectopic pregnancy without laparotomy: Defining the incidence of manageable patients. Int J Gynecol Obstet 1988;27:247.

4. Bonatz G, Lehmann-Willenbrock E, Kunstmann P, Semm I, Hedderich J, Semm K. Management of patients with persistent beta-hCG values following laparoscopic surgical and local drug treatment for ectopic pregnancy. Int $\mathbf{J}$ Gynecol Obstet 1994;47:33.

5. Lundorff $\mathbf{P}$, Hahlin M, Sjoblom $\mathrm{P}$, Lindblom B. Persistent trophoblast after conservative treatment of tubal pregnancy: Prediction and detection. Obstet Gynecol 1991;77:129.

6. DiMarchi JM, Kosasa TS, Kobara TY, Hale RW. Persistent ectopic pregnancy. Obstet Gynecol 1987;70:555.

7. Rose PG, Cohen SM. Methotrexate therapy for persistent ectopic pregnancy after conservative laparoscopic management. Obstet Gynecol 1990;76:947.

8. Hoppe DE, Bekkar BE, Nager CW. Single dose systemic methotrexate for the treatment of persistent ectopic pregnancy after conservative surgery. Obstet Gynecol 1994;83:51.

9. Vermesh M, Silva P, Sauer MV, Vargyas J, Lobo RA. Persistent tubal ectopic gestation: Patterns of circulating $\beta$ human chorionic gonadotropin and progesterone, and management options. Fertil Steril 1988;50:584.

10. Cowan BD, McGhee RP, Bates GW. Treatment of persistent ectopic pregnancy with methotrexate and leucovorin rescue. A case report. Obstet Gynecol 1986;67:50(S).

11. Sauer MV, Gorrill MJ, Rodi IA, et al. Nonsurgical management of unruptured ectopic pregnancy: An extended clinical trial. Fertil Steril 1987;48:752.

12. Carson SA, Buster JE. Ectopic pregnancy. N Engl J Med 1993;329:1174.

Address reprint requests to:

Mark V. Sauer, M.D.

Columbia Presbyterian Medical Center

Division of Reproductive Endocrinology

622 West 168th Street, PH 16-28

New York, NY 10032-3784 